\title{
In Situ TEM-EELS for Tracking Lithium Reactions in Battery Electrodes
}

\author{
Feng Wang, Sung-Wook Kim, Lijun Wu, Dong Su, Yimei Zhu, and Jason Graetz \\ Brookhaven National Laboratory, Upton NY 11973 USA
}

Lithium ion batteries have become the most popular rechargeable storage devices for portable electronics, and are leading candidates of power sources for electrical vehicles and grid storage. In order to design safe, high-energy electrodes with long cycling life, we need a better understanding how electrode materials function by real-time tracking of lithium transport and electrochemical reaction in a working electrode, ideally at the level of single particles. This type of characterization also requires high sensitivity to $\mathrm{Li}$ and other constituents at relevant spatial and temporary resolution, along with the capability of capturing changes of electrode materials in both electronic and atomic structure. High-resolution transmission electron microscopy (TEM) imaging and energy-loss spectroscopy (EELS), have been shown powerful for local structural analysis, with high sensitivity to Li and sub-nm spatial resolution at the optimized operation conditions [1], but they have been mostly used for ex situ measurements, providing limited information about phase nucleation and propagation, lithium transport and reaction kinetics of electrodes. The development of an electrochemical cell for in situ TEM studies has remained elusive due to the limited space between the objective lens pole pieces and critical requirement for electron transparency.

We have recently custom-built an electrochemical cell to operate inside the TEM, providing exquisite, real-time live images of individual nanoparticles in a working battery electrode (Figure 1a) [2]. A suite of in-situ techniques, including high-resolution imaging, EELS and electron diffraction have also been developed for obtaining comprehensive information on structural and chemical evolution during lithium reaction process, and used for studies of conversion reaction in iron fluoride $\left(\mathrm{FeF}_{2}\right)$, a potential cathode with 2-3 times higher capacity than today's intercalation electrodes [2,3]. These real-time experimental observations, supported by advanced computation, revealed that the lithium ions swept rapidly across the surface of the nanoparticles in a matter of seconds. The transformation then moved slowly through the bulk in a layer-by-layer process that split the compounds into distinct regions (Figure 1b). This study represents an important step forward in our understanding of electrochemical conversion reactions and the role of electron and ion transport in the formation and evolution of the nano-structure in electrodes. This method has been also applied to measure the lithium propagation across a thin $\mathrm{FeF}_{2}$ film (Fig. 1c, d) and track the chemical and structural changes in multiple particles during conversion process (Figure 2). The versatility of this new approach to in-situ TEM studies will be discussed along with our recent results on a variety of systems including conversion electrodes $\left(\mathrm{FeF}_{2}, \mathrm{FeOF}\right)$, alloying electrodes $\left(\mathrm{Si}, \mathrm{SnCo}_{\mathrm{x}}\right)$ and intercalation electrodes $\left(\mathrm{Li}_{4} \mathrm{Ti}_{5} \mathrm{O}_{12}\right)[4,5]$.

\section{References}

[1] F. Wang, et al. "Tracking Lithium Transport and Electrochemical Reactions in Nanoparticles." Nature Communications 3, 1201(2012).

[2] F. Wang, et al. "Conversion Reaction Mechanisms in Lithium Ion Batteries: Study of the Binary

Metal Fluoride Electrodes”, J. Am. Chem. Soc., 133, 18828, (2011).

[3] F. Wang, et al., "Chemical distribution and bonding of lithium in intercalated graphite: Identification with optimized electron energy loss spectroscopy”, ACS Nano, 5, 1190 (2011).

[4] D. Su, et. al., "Engineering nano-composite Li4Ti5O12 anodes via scanning electron-probe fabrication", Adv. Energy (in press).

[5] Work supported by the U.S. DOE under contract DE-AC02-98CH10886 with funding from DOE/BES-EFRC-NCCES. 

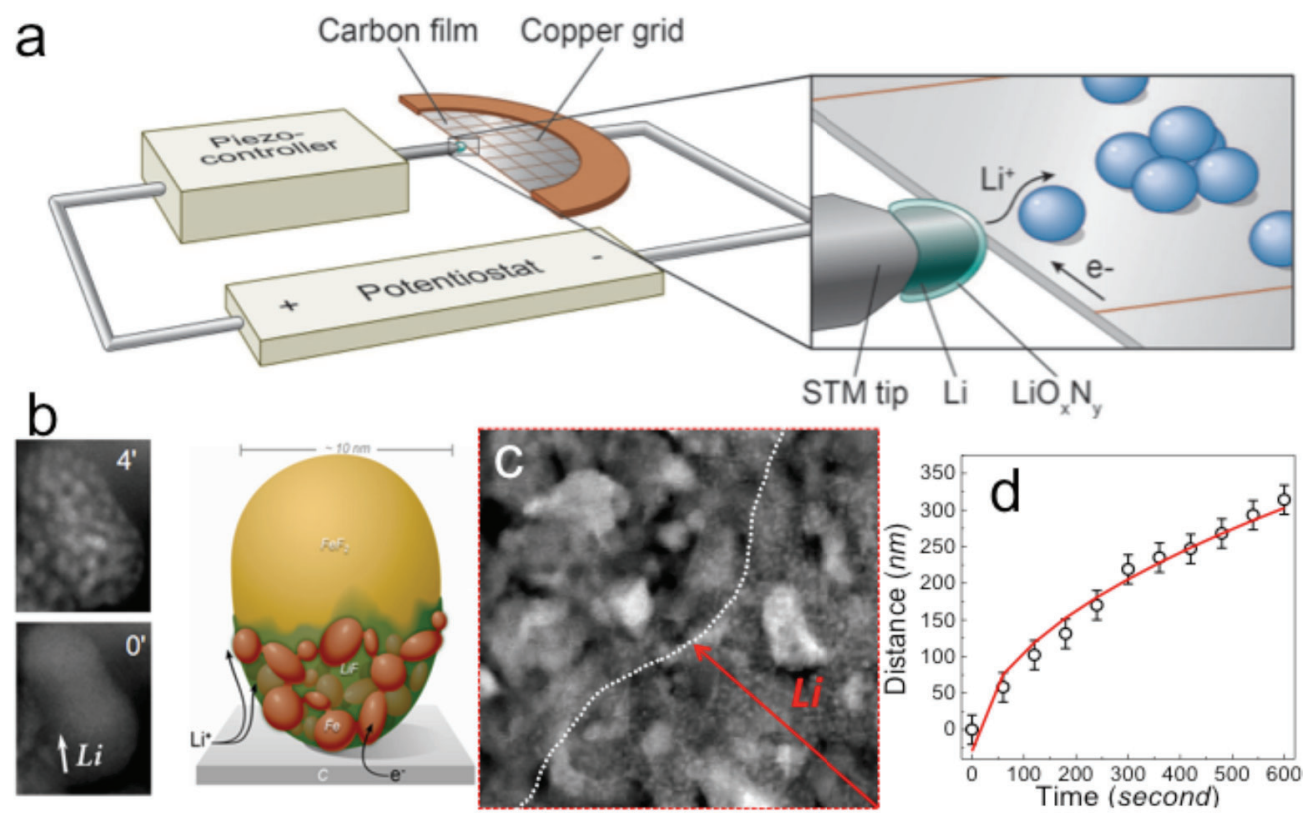

Figure 1. (a) schematic of the in-situ electrochemical cell developed for TEM; (b) Time-lapse annual dark-field STEM images of $\mathrm{FeF}_{2}$ nanoparticles, and illustration of the propagation of the reaction front through a single particles via "layer-by-layer" process and possible pathway for electron and $\mathrm{Li}^{+}$transport; (c) propagation of lithium across the $\mathrm{FeF}_{2}$ film with time (c), and distance as a function of time, fit with a power flaw, $\sim \mathrm{t}^{1 / 2}$, indicating an lithium-diffusion dominated transportation mechanism.
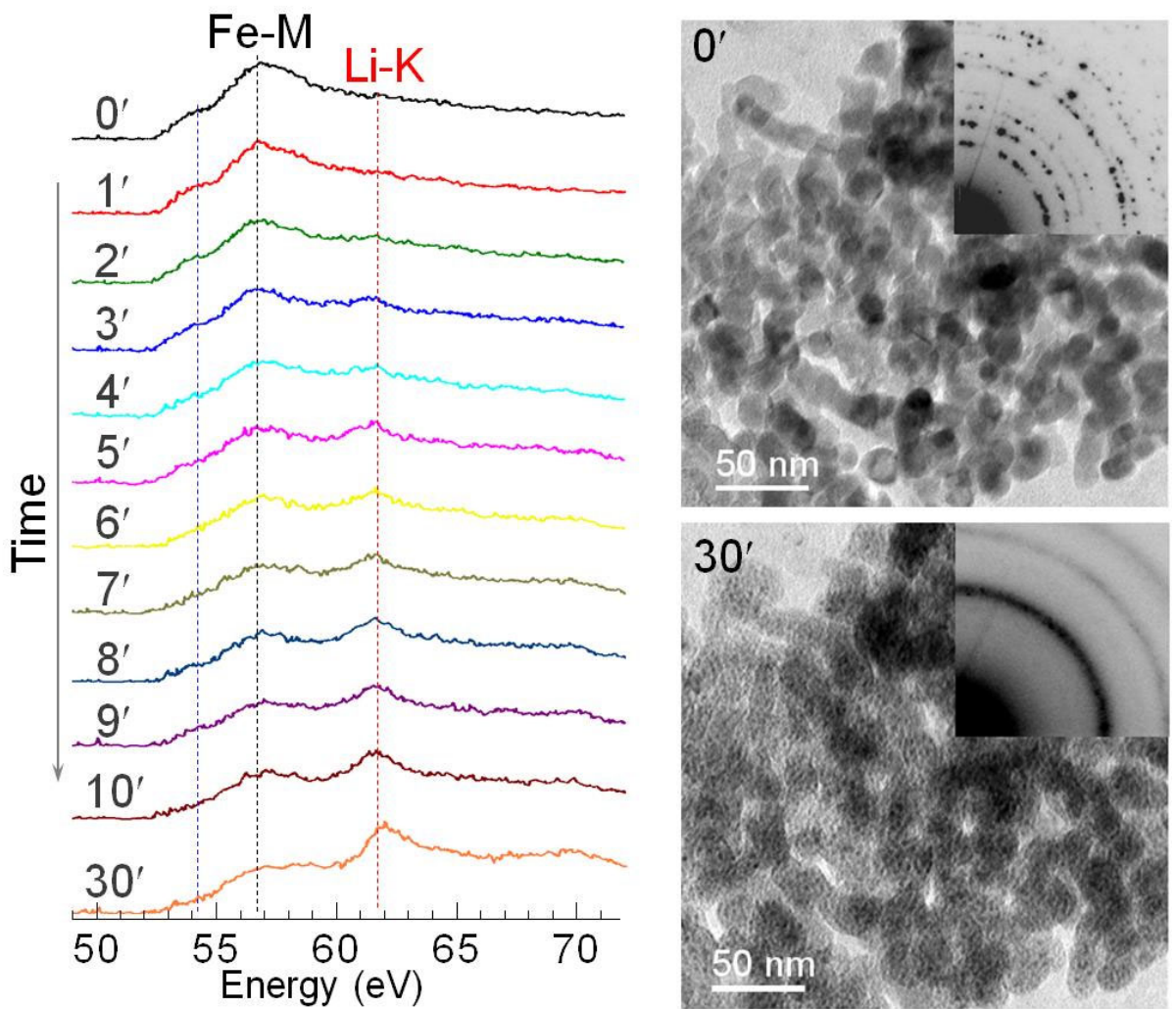

Figure 2. In situ EELS by recording thousands of spectra from $\mathrm{FeF}_{2}$ particles, for tracking chemical and structural changes by fine structure of $\mathrm{Fe} \mathrm{M}$ - and $\mathrm{Li} \mathrm{K}$ - edges during conversion process: $\mathrm{FeF}_{2} \rightarrow \mathrm{LiF}+\mathrm{Fe}$, coupled with bright-field TEM images (right), electron diffraction patterns (inset). 